\title{
OPTIMIZING THE NETWORK FLOW IN CLOUD SUPPLY CHAIN MANAGEMENT
}

\author{
Partha Sarathi Pattnayak ${ }^{1}$, Jayram .Pradhan ${ }^{2}$ and Sudhanshu Shekhar patra ${ }^{3}$
}

\begin{abstract}
To deploy and operate ICT based services organization using cloud computing as new computing paradigm. This computing concept initiate many benefits like large scale of flexibility, pay-as-per use models, the supply prerequisites can be done rapidly and high degree of resource availability compared to the traditional data centers. However, as there is no such definite SC in support of clouds plus linked information models are available, it is restraining the acceptance of CSC (cloud supply chain). This paper initiates the CSC, which renders consumers plus providers of the CSC to undoubtedly define their place inside supply chain. The management of SC is getting more and more attention in a wired world demanding fast response and flexibility with excellent service quality. There are, as there should be, many studies done to aid companies with better knowledge in operating their supply chains. However, these studies are either too conceptual to be directly put into use, or too specific and complex to be applied to an individual case. The main objective of this paper is to find out the maximum flow given Supply chain network (SCN) plus product tree structure.

Keywords: Supply chain Management, Cloud Computing, Max Flow, Flow network, EPC
\end{abstract}

\section{INTRODUCTION}

The management and control of all materials and information in the logistics process is interpreted the same as Supply chain management (SCM). It begins with the attainment of raw materials ends with the release to the end user. To gain an edge in a highly competitive market environment, enterprises are constantly engaged in discovering new tools and methods to control their costs and make fast and correct decision in order to increase their profit. The focus is to improve product quality, product time compression and costs and lead-times reduction. These goals can only be realized through well-planned supply policy which is termed as supply chain policy (SCP) or SCM. The Supply Chain (SC) thus can be defined as an integrated approach of physical handling of goods and virtual information from suppliers to customers. Supply chain manifests a system of organization that involves people, information and resources. It includes a process that moves a product from supplier to customer (figure 1).Supply Chain management is considered indispensable for a competitive market as it helps in delivering particular goods in perfect time and manner at the appointed place where it is to be reached.

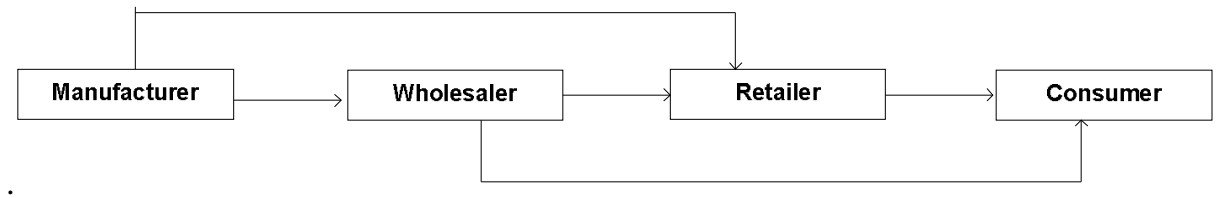

Figure 1 : Retail Supply Chain

The firms create the supply chain jointly from end to end physical flows and information flows. Transformation, movement, and storage and materials are consider as physical flow and most visible piece of supply chain (SC). The

\footnotetext{
${ }^{1}$ School of Computer Application, KIIT University, Bhubaneswar, India

${ }^{2}$ Department of Computer Science, Berhampur University, Berhampur, India

${ }^{3}$ School of Computer Application, KIIT University, Bhubaneswar, India
} 
different SC associates to manage their continuing policy, and to organise routine flow of merchandise up and down the supply chain. Continual improvement of supply chain management has been a key focus in many organizations leading to significant cost savings. This study examines to find out the utmost or maximum flow stand on the certain SCN (supply chain network) and merchandise point collection. Recently, companies are looking into ways to maximize cost and operation efficiency of each stage of supply chain. It covers planning, forecasting, logistics, sourcing, procurement and management of spare parts etc. Modern technology makes it easy for the organization to access to information in their respective fields while helping the activities to be coordinated to manage the supply chain. Besides optimizing customer value, it also helps in sustaining competitive advantage. Supply chain activities includes product development, sourcing, production, logistics and the information systems required to coordinate these activities. The technology of cloud computing is useful for optimization as it provides platform, infrastructure and software solutions for supply chain through internet. The services based on clouds when utilized in management of supply chain, brings in various benefits such as low cost in comparison to infrastructure cost, platform scalability, visibility of supply chain. It also promotes flexibility by striking collaboration among partners of supply chain. Cloud computing refers to distributed computing, networking, virtualization and web services. Cloud consists of different aspects like data centre, clients and distributed servers. It also talks about scalability, flexibility and fault tolerance. It also reduces the cost of ownership and provides on demand services etc.

Simplification constitutes the advantage system based on cloud. By using same type of platform the cloud can eliminate the problem of compatibility. Every part of supply chain is connected by the cloud. It collaborates supply chain information between partners in one supply chain system, it configurates information related to supply chain among partners. The supply chain members get into cloud's collaborative environment by using member id and password. All users are given power in the same platform to operate which would result in reducing the time of response by supply chain partners. Visibility is the benefit that helps the different participants of supply chain to connect in time. It helps companies to manage various customers to coordinate their operations .It also facilitates the whole system can be viewed in a transparent way through customer network. The real time inventory visibility and shipments are provided by systems based on cloud. It also facilitates in tracking of logistics. Through the computation of cloud, companies can control their system capacity very accurately. During the period of high demands, companies require enough capacity to tackle the increasing demand. The common on-field systems help the companies to posses the required database for the whole year to tackle more demands in a short span of time. The technology of cloud makes the companies to adjust their respective capability automatically as per their requirement besides scaling their computing power based on changing demands.

The development of information technology results in matured transmission technology of internet network while improving various fields of security and stability. It strikes compatibility by continuous expanding the range of application continuously. Cloud is very much flexible. It possess the ability to make the computing power adaptable to the requirement of the users. This word is known as scalability. Scalability ensures the availability of service of computing to the users at any point of time. It is a crucial issue in the management of supply chain. Supply chain is distributed in nature. The firm needs to grow its supply and distribution system that results in to scale IT services of supply chain to a great extent. In the case of any necessity, cloud provides services to the supply chain users as per their demands. If a firm has a global distribution of supply chain then it calls for a unique cloud infrastructure for its individual branch. Company's own private cloud system can provide secure information that shared among different users of supply chain. Firms using supply chain have different branches located in different parts of the world. Thus, the companies are dependent on both centralized cloud data center and distributed data center as well. Cloud computing refers to a standardized Information technology that provides service like software application through Internet. Cloud consists of three parts namely, Infrastructure as a Service, Software as a service and Platform as a service. Companies spend a lot to develop their own software system. The adoption of IT model aid that lesser their TCO if match up towards established on top of and off principle data centre models [3], [9]. A single data centre served masses and variety of customers and functions by the means of communications which wires cloud computing augment the customisation, suppleness with capability of resource acquirement, practice plus continuation [2], [7]. On the other hand several organisations are uncertain to hold cloud computing, still they believe it to be a workable representation [7], [10]. Due to the feeling of uncertain behaviour which is a potential cause concerning the impact of adjust on the whole Information Technology setting and functions [4], [9]. In conventional IT solutions for example, the customer opts for buying both operating systems and servers as well. It makes the concerned customer to spend a lot to install one's own hardware and also for its maintenance. In contrast, the modern cloud solution gives the option to subscribe the required software services only. There is no need of buying own hardware and software and managing the human capital. The customer has the option to select and use according to one's own requirement. The problem area offers a pertinent solution to the current supply chain management across the globe. While the stakeholders use various expensive infrastructures to address the issue the 
new mechanism offers the better option with minimum cost for the supply chain management. Electronic Product Code tag and upload in federal worldwide database which is stored at cloud (figure 2). Here, EPC code acts as an input for the product. This information can be accessed by the customers. The logistic one can keep the track of the products with EPC tag when the product is in transit. CLOUD based infrastructure helps in reducing the IT expenditure as it rules out the purchase of unnecessary software and hardware. It converts it into a centralized Hardware and Software[6]. While the EPC is connected with managing the goods on shelf and billing in retail specter, in warehouse, the goods are classified to be handed over to the traders.

This paper is introduced the cloud supply chain to overcome the gap in technical hurdles, loss of control, that is psychological effects, be short of complete impression of the entire supply chain and lost close into information flow, check prerequisites plus method of book-keeping and cost of cloud services to solve the hinders companies from implementation the cloud.

\section{THE CLOUD SUPPLY CHAIN}

\section{Definition:}

The introduction of SC in the framework of cloud computing is relatively modern plus release up a recent study. Generally, SC involves more than two parties those are connected by a network of goods, information, and funds [8]. When supply chain is applied in cloud computing one can notice the following deviations: In Cloud Supply Chain (CSC), more than two parties are joined in terms of cloud services allied information plus stocks. According to Cachon and Fisher [1], apart from the cost of information sharing inside SC, the cost is also incurred while restructuring of services and the management, information, and funds. The cost of optimization of the chain is also included in the CSC. A SC [5], focuses on two types of functions namely, Physical Function and Market mediation function. A physical function involves production of goods out of raw material / intermediate parts / components and transport of goods to the appointed location. The Market mediation function highlights that different products those reach the market place must cater in to the demands of the customers.

The physical function is important for functional products. The Market negotiation task is crucial than the physical utility as it is necessary for new products. [8].The unique features of CSC is to provide the software services which is included in the physical function and presenting the services with a modular design that is highlighted by the function of market mediation.

\section{Cloud Supply Chain Components:}

Primarily, the CSC of cloud services requires to be identified. Then it has to be run plus controlled from both technical with business perspectives. The CSC focuses a group of interrelated businesses in the area cloud computing. It also takes care of the combined packages those are needed by customers as end CS. Thus, SC for the cloud is entrusted with the partial functions of coordinating plus controlling the bi-directional progress of information, stocks and services. It looks in to various matters like facilitating the actual infrastructure services, monitoring of services by making provisions for the information processes plus virtual machines. It supports both billing plus accounting processes. On the way to make the work more effective within a complex supply chain system, it is a prerequisite to identify and define the services exchanged plus actors (products along the cloud supply chain), the run in stock plus information. (Figure 3).

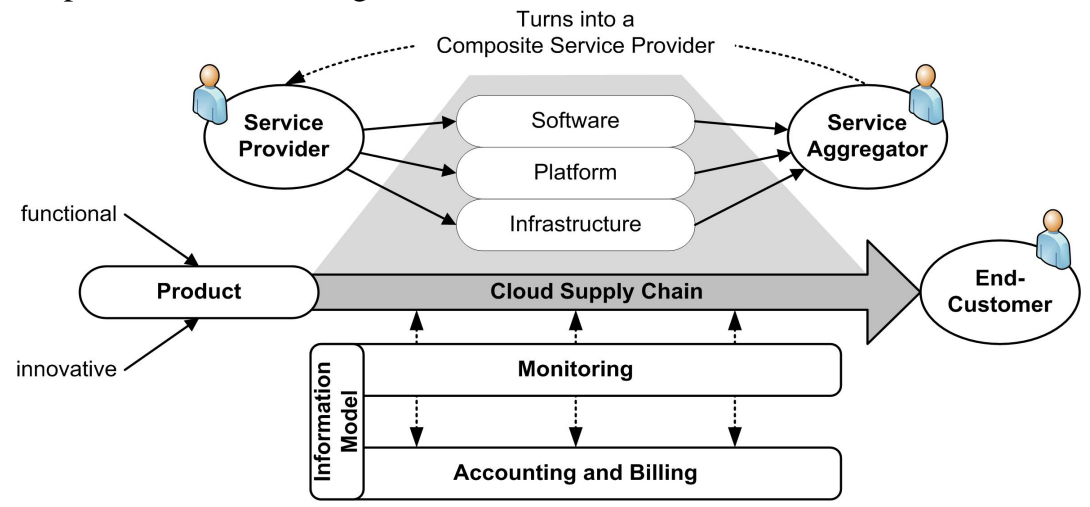

Figure 3 : Cloud Supply Chain 
Main Actors - A number of performer have to be recognized next to the SC. Service contributors are required to adopt several roles within the CSC. They are expected to act as infrastructure, platform or software providers. They also need to be directly get in touch with the end-consumer. Sometimes, they take a role of a broker (actor service provider) or a trade partner of a service aggregators, so as to utilize the supply service. It can also create a composite service by combining the services. For an example, a complex provision can be a part of $\mathrm{s} / \mathrm{w}$ that works as a service on crest of a flexible supply platform. Thus, the manufactured goods for the end-consumer is to provide SAAS in flexible term. To maintain visibility and transparency for monitoring, accounting and billing, SAAS becomes more indispensable when a particular supply network is organised. Transparency is very much necessary as an endproduct can easily be turned in to a complex process while involving a number of actors.

\section{RELATED WORK}

Two categories are used to classify related studies, namely, conceptual-oriented and case-oriented studies. The differentiation line is drawn at how a study presents and generalizes its result. A study is said to be a conceptual oriented one if the stress of the study is on the instructions of general concepts, while the study can result from a real world case. On the contrary, a case-oriented study focuses more on some special cases and the experiences learnt. Ambiguity exists as it should, but the distinction is still clear.

Lee and Billington [17], Billington [12] and Fisher [16] are examples of conceptual-oriented studies. In this sense, they all introduced many great concepts in managing the supply chain or the inventory in a supply chain.However, the lack of detail description of how to build one's own model may disappoint some managers. For example, Lee and Billington [17] were quite descriptive with many illustrative examples to clarify their point of view. In the article, fourteen often seen pitfalls were discussed, including the basic misunderstanding and misusage of supply chain, the operational problems encountered, and some strategic issues. There were also some opportunities observed by them worth noticing. Fisher [16] provided a good grid to identify the fitness of the product of a company and its supply chain. While it is very helpful, a manager may want to know more about how to find some details in his own situation. On the other hand, Arntzen et al. [11], Lee and Billington [18] and Cohen and Mallik [15] serve as good examples of case-oriented studies. Experiences were illustrated and can be learnt for those companies who have the capabilities or are ready to start building such models. However, medium to small companies may not benefit much from these studies, since small companies wouldn't afford to build complex mathematical models. Besides, the formulations may not apply to specific condition. There are still researches trying to provide more general yet mathematical help. Chen and Chern [13], [14] have done some studies utilizing the network flow algorithms such as shortest path algorithm and maximum flow algorithm. In their studies, a general supply chain graph, with the graph of the production tree of the supply chain, is transformed into another form that is ready to apply the shortest path or the maximum flow algorithm. With each algorithm, their algorithms are suitable for easy solution about the lowest cost production path or the maximum amount of production in the supply chain. Hence each of their algorithms provides a simple yet mathematical way for supply chain managers to make decisions regarding one of the above issues. However, their model works for either lowest cost or maximum production output only, but it is usually necessary to consider both objectives in a supply chain decision. Therefore, it is possible to improve their studies by combining the two considerations. According to the above reviews, it is obvious that there is a need for a general but mathematical model for taking into account of cost and production amount. This study, based on the results achieved by Chen and Chern [4, 5], tried to answer the call with the following algorithm that provides such a solution

\section{MODEL FORMULATION}

The algorithm of the maximum production output with the lowest cost consideration in a SC works as follows. First the graph of the SC is transformed to live to apply any of the traditional maximum flow algorithms. The material moves through the network in a flow (rate) and different directed edge be a passage for the goods by means of various distinct capacity. Nodes of the graph are the connection points which never collects material i.e., thematerials reaching into a node must be equal to the flow of material leaving the node, known as the flow of conservation.

\subsection{Maximum Flow Concept}

The max flow-min cost "path" found in a network is a route that includes necessary entities to produce the final product. Further, the minimum "cost" is not necessarily the financial cost incurred, but it may be expressed in time, actual distance or other relating factors from a source node to a sink node. So it can be suitably used in a supply chain to find the lowest cost. It represents the critical path in the supply chain. A manager can arrange the allocation 
of resources/orders in his supply chain as per the critical path. The model itself is general and easy to build without the investment of a large amount of money or professional knowledge. It is considered important especially for small to middle size businesses in Asian region as it is impossible for these companies to hire or introduce complex modeling staff or technique to enhance their management in supply chains. Secondly, with the help of this algorithm, the model can produce an initial result of the possible path to generate the products required in a supply chain. It is especially important when there is no time to evolve complex models while the market are required to respond to the demands in a quicker way. It is also required for the managers when they want to get an approximate yet quantitative idea about the shortest production cycle or the lowest cost possible to produce a product in their supply chains. Lastly, as the model is split in a "product tree" fashion to apply the shortest path algorithm, different "sections" or "cuts" can be noticed existing in the supply chain. It can also be observed that these levels have some relationships with the cuts in the supply chain. These levels are noticed in hierarchical positions in the supply chain networks. It is to find a way to optimize in each cut to achieve some degree of optimal solution for the whole supply chain. This phenomena is worth studying for the researchers or managers. By looking in to their own supply chain models, companies / firm may achieve some great insight for better understanding and management of their supply chains. The material is produced at the source vertex s, and the sink node is $t$ where the material is consumed. For all other nodes what materials goes in is equal to what materials goes out, known as the flow of conservation. The aim of the problem is to compute the maximum rate of material flow from source $\mathrm{s}$ to sink $\mathrm{t}$.

\subsection{Formal Definition of Max Flow Problem}

i) Consider a graph $\mathrm{G}=(\mathrm{V}, \mathrm{E})$ is a flow network, where $\mathrm{V}$ is the set of vertices and $\mathrm{E}$ is the set of edges.

- $\quad$ Graph $\mathrm{G}$ is a directed graph where all the edge $\mathrm{e}=(\mathrm{v} 1, \mathrm{v} 2) € \mathrm{E}$ has a positive capacity $\mathrm{c}(\mathrm{v} 1, \mathrm{v} 2)>=0$

- $\quad$ There are two special vertices : source $s$ and sink t.

- $\quad$ For any vertex $v$, there is a path $s->\ldots . .>\mathrm{v} . . .->t$

- If an edge $(\mathrm{v} 1, \mathrm{v} 2) € \mathrm{E}$, then there should n't be an $(\mathrm{v} 2, \mathrm{v} 1)$ in the reverse direction.

- $\quad$ The graph shouldn't have self loops.

- If there exists no edge $(\mathrm{v} 1, \mathrm{v} 2) € \mathrm{E}$, we define $\mathrm{c}(\mathrm{v} 2, \mathrm{v} 1)=0$

ii) Flow, a function $f: V \times V \rightarrow R$

Capacity Constraint $: \forall \mathrm{v} 1, \mathrm{v} 2 € \mathrm{~V}: \mathrm{f}(\mathrm{v} 1, \mathrm{v} 2)<=\mathrm{c}(\mathrm{v} 1, \mathrm{v} 2)$

skew symmetry: $\forall$ v1 , v2 $€ \mathrm{~V}: \mathrm{f}(\mathrm{v} 1, \mathrm{v} 2)=-\mathrm{f}(\mathrm{v} 2, \mathrm{v} 1)$

Flow Conservation: $\forall \mathrm{v} 1 € \mathrm{~V}-\{\mathrm{s}, \mathrm{t}\}: \sum_{v 2 \epsilon v} f(v 2, v 1)=\sum_{v 2 € v} f(v 1, v 2)$

When there is no edge $(\mathrm{v} 1, \mathrm{v} 2)$ in $\mathrm{E}$, there can be no flow from $\mathrm{v} 1$ to $\mathrm{v} 2$, and $\mathrm{f}(\mathrm{v} 1, \mathrm{v} 2)=0$.

A flow network $\mathrm{G}=\langle\mathrm{V}, \mathrm{E}\rangle$ is shown in figure 4. A flow in $\mathrm{G}$ with value $|f|=5$. Each edge $(\mathrm{v} 1, \mathrm{v} 2)$ is labeled by $\mathrm{f}(\mathrm{v} 1, \mathrm{v} 2) / \mathrm{c}(\mathrm{v} 1, \mathrm{v} 2)$.

4.3 Flow cancellation

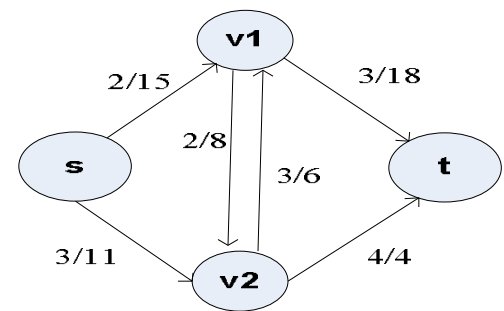

Figure 4 : Flow Concepts

Using skew symmetry, within the same pair of vertices two positive flows in opposite directions could be cancelled ( may be partially). This is shown in figure 5 between the pair of vertices v1 and $\mathrm{v} 2$.

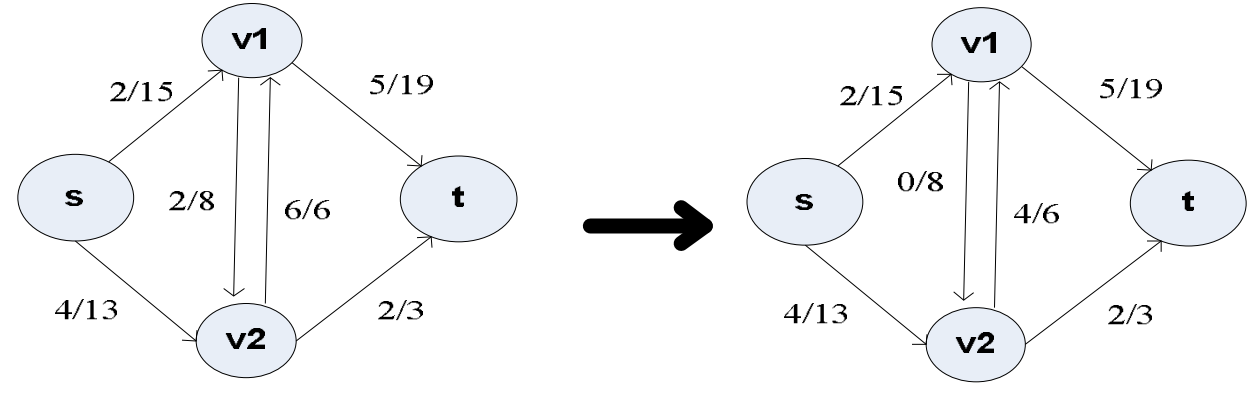


Figure 5 : Cancellation of Flows

The method Ford-Fulkerson is an iterative method. We initialize with $\mathrm{f}(\mathrm{v} 1, \mathrm{v} 2)=0$ for all $\mathrm{v} 1$, v2 $€ \mathrm{~V}$. In every iteration, find an "augmenting path" and increase the flow value with this, through more flow can be send, and then augmenting the flow along this path. The process will be repeated till there no augmenting path is found. On termination the max-flow min-cut theorem shows that, the process gives a maximum flow.

step 0 : Find a directed path from source $s$ to sink $t$ in the graph $\mathrm{G}$.

step 0': Find the minimum capacity b on the path .

Assign $R(e)=b$ for all e on the path.

General Step : 1. Build a residual graph $\mathrm{G}_{\mathrm{f}}$, given $\mathrm{F}$.

i) For each edge $e=(v 1, v 2)$ with $\mathrm{R}(\mathrm{e})>0$ as shown in figure 6 , direct an edge

from $v 2$ to $v 1$ with residual capacity $=R(e)=c^{\prime}(e)$ shown in figure 7 .

ii) For $\mathrm{e}=(\mathrm{v} 1, \mathrm{v} 2)$ with $\mathrm{R}(\mathrm{e})<\mathrm{C}_{\mathrm{e}}$ as shown in figure 8 , put a forward edge from

v1 to v2 with $C^{\prime}(e)=C_{e}-R(e)$ as in figure 9.

2. Find an s-t directed path in $\mathrm{G}_{\mathrm{f}}$ if there is one else stop and let $\mathrm{b}$ be the minimum residual capacity on $\mathrm{P}$.

3. Augment $f$ using $P$ and $b$.

i) Increase the flow of the network if e be a forward edge in path $P$.

$$
R^{\prime}(e)=R(e)+b \text {. }
$$

ii) Decrease the flow of the network if e be a backward edge in path $P$.

$R^{\prime}(e)=R(e)-b$

4. Repeat steps 1-3 till no augmenting path exists.

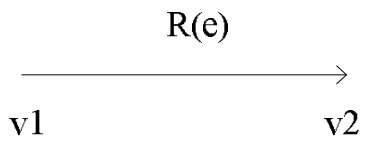

Figure 6 : Edge $e=(v 1, v 2)$ with $R(e)>0$

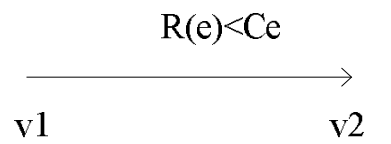

Figure 8: Edge e=(v1,v2) with $\mathrm{R}(\mathrm{e})<\mathrm{Ce}$

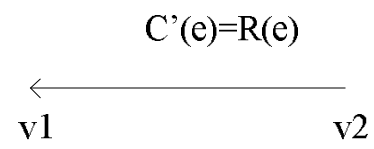

Figure 7 : If $\mathrm{R}(\mathrm{e})>0$ in $\mathrm{G}$, then in the residual graph draw an edge (v2,v1) with $C^{\prime}(e)=R(e)$

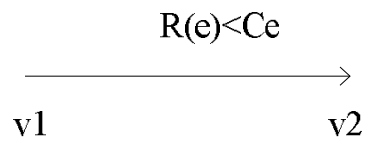

Figure 9: If $\mathrm{R}(\mathrm{e})<\mathrm{Ce}$ in $\mathrm{G}$, then in the residual graph draw an edge (v1,v2) with Ce-R(e)

\section{FORD-FULKERSON-METHOD}

\section{$(\mathrm{G}, \mathrm{s}, \mathrm{t})$}

$\% \%$ Input : Graph $\mathrm{G}$

$\% \%$ Output : Max-flow

1. Initialization: For every edge e€ E do

2. flow $\mathrm{f}=0$

3. End for

4. While (there exists an augmenting path $\mathrm{P}$ in residual graph) do

5. Find min-flow in an augmenting path $\mathrm{P}$
6. For all $(\mathrm{v} 1, \mathrm{v} 2)$ of $\mathrm{P}$ do

7. Update min-flow using $P$ and modify the residual network

8. End for

9. End while

10. Return max-flow 


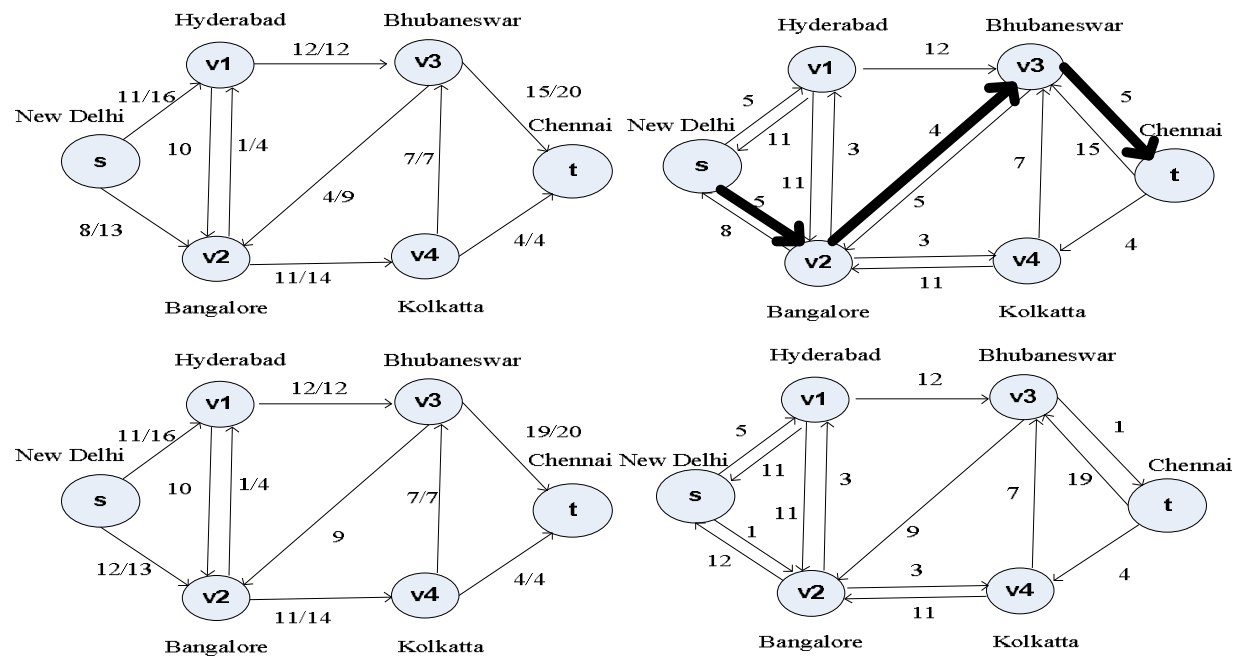

Figure 10 (a) Graph $G$ is the flow network (b) The shaded portion is the residual network $G_{f}$ with augmenting path $P$. Its residual capacity $=c_{\mathrm{f}}(p)$ $=\mathrm{c}\left(\mathrm{v}_{2}, \mathrm{v}_{3}\right)=4$. (c) The flow in $\mathrm{G}$ results by augmenting along path $\mathrm{P}$ by unit 4 , the residual capacity. (d) The residual network generated by the flow in (c). As there will be no augmenting path the maximum flow will be $=11+12=23$.

\section{CONCLUSION}

The algorithm discussed here is a step toward a general model of a supply chain management problem. There are some points worth considering in the study. First of all, the result yielded from the algorithm serves as an initial basis for a manager to make a decision about saving the most from his supply chain so as to fulfil the demand of the market. Since the market is greatly fluctuant, sometimes a manager needs to make a quick decision. The algorithm developed herein is simple enough to assist the job.

\section{REFERENCES}

[1] Cachon, G., Fisher, M: Supply chain inventory management and the value of shared information. Management Science, Vol. 46 No. 8 (2000), 1032-1048.

[2] C. C. Centre. Moving from on premise to the cloud - the importance of partnership, (2010).

[3] C. C. Centre. Solutions in the cloud vs on-premise software solutions (2010).

[4] Feiman, J., Cearley, D.W.: Economics of the cloud: Business value assessments, (2009).

[5] Fisher, M. What is the right supply chain for your product? Harvard Business Review, (1997), 105-116

[6] E. E. Group. The future of cloud computing - opportunities for european cloud computing beyond 2010

[7] Li, X., Li, Y., Liu, T., Qiu, J., Wang, F.: The mothod and tool of cost analysis for cloud computing. IEEE International Conference on Cloud Computing ( 2009), 93 -100.

[8] Paulitsch, M.: Dynamic Coordination of Supply Chains. PhD thesis, (2003).

[9] Research. G. Hype cycle for cloud computing, (2009).

[10] Roehrig, P., Ferrusi, C., Shanahan, A.: Major hurdles remain in enterprise cloud services - IT service providers are addressing the technical and business challenges for end users, (2009).

[11] Arntzen, B. C., Brown, G. G., Harrison, T. P., \&Trafton, L. L. (1995). Global supply chain management at Digital Equipment Corporation. Interfaces, 25(1), 69-93.

[12] Billington, Corey: Strategic Supply Chain Management, OR/MS Today (1994), 20-27.

[13] Chen, Shu-Yi, and Ching-Chin Chern, "Shortest Path for A Supply Chain Network," Proceedings of the 4th Asia Pacific Decision Sciences Institute Conference, Shanghai, China, (1999), 579-582.

[14] Santoso, T., Ahmed, S., Goetschalckx, M., \& Shapiro, A. (2005). A stochastic programming approach for supply chain network design under uncertainty. European Journal of Operational Research, 167(1), 96-115.

[15] Cohen, M. A., \&Mallik, S. (1997). Global supply chains: research and applications. Production and Operations Management, 6(3), 193210 .

[16] Fisher, Marshall L., "What Is the Right Supply Chain for your Product?” Harvard Business Review (1997), 105-116.

[17] Lee, Hau L., and Corey Billington, Managing Supply Chain Inventory: Pitfalls and Opportunities, Sloan Management Review, spring (1992), 65-73.

[18] Lee, Hau L., and Corey Billington. "Material management in decentralized supply chains." Operations research 41, no. 5 (1993): 835-847. 